\title{
A New Attack on the LEX Stream Cipher
}

\author{
Orr Dunkelman ${ }^{1, \star}$ and Nathan Keller ${ }^{2, \star \star}$ \\ ${ }^{1}$ École Normale Supérieure \\ Département d'Informatique, \\ CNRS, INRIA \\ 45 rue d'Ulm, 75230 Paris, France \\ orr.dunkelman@ens.fr \\ ${ }^{2}$ Einstein Institute of Mathematics, Hebrew University \\ Jerusalem 91904, Israel \\ nkeller@math.huji.ac.il
}

\begin{abstract}
In 6], Biryukov presented a new methodology of stream cipher design, called leak extraction. The stream cipher LEX, based on this methodology and on the AES block cipher, was selected to phase 3 of the eSTREAM competition. The suggested methodology seemed promising, and LEX, due to its elegance, simplicity and performance was expected to be selected to the eSTREAM portfolio.

In this paper we present a key recovery attack on LEX. The attack requires about $2^{36.3}$ bytes of key-stream produced by the same key (possibly under many different IVs), and retrieves the secret key in time of $2^{112}$ simple operations. Following a preliminary version of our attack, LEX was discarded from the final portfolio of eSTREAM.
\end{abstract}

Keywords: LEX, AES, stream cipher design.

\section{Introduction}

The design of stream ciphers, and more generally, pseudo-random number generators (PRNGs), has been a subject of intensive study over the last decades. One of the well-known methods to construct a PRNG is to base it on a keyed pseudo-random permutation. A provably secure construction of this class is given by Goldreich and Levin [19. An instantiation of this approach (even though an earlier one) is the Blum and Micali [1] construction (based on the hardness of RSA). A more efficiency-oriented construction is the BMGL stream cipher [21] (based on the Rijndael block cipher). However, these constructions are relatively slow, and hence are not used in practical applications.

\footnotetext{
* The first author was supported by the France Telecome Chaire. Some of the work presented in this paper was done while the first author was staying at K.U. Leuven, Belgium and supported by the IAP Programme P6/26 BCRYPT of the Belgian State (Belgian Science Policy).

** The second author is supported by the Adams Fellowship Program of the Israel Academy of Sciences and Humanities.
} 
In [6], Biryukov presented a new methodology for constructing PRNGs of this class, called leak extraction. In this methodology, the output key stream of the stream cipher is based on parts of the internal state of a block cipher at certain rounds (possibly after passing an additional filter function). Of course, in such a case, the "leaked" parts of the internal state have to be chosen carefully such that the security of the resulted stream cipher will be comparable to the security of the original block cipher.

As an example of the leak extraction methodology, Biryukov presented in 6 the stream cipher LEX, in which the underlying block cipher is AES. The key stream of LEX is generated by applying AES in the OFB (Output Feedback Block) mode of operation and extracting 32 bits of the intermediate state after the application of each full AES round.

LEX was submitted to the eSTREAM competition (see [7]). Due to its high speed (2.5 times faster than AES), fast key initialization phase (a single AES encryption), and expected security (based on the security of AES), LEX was considered a very promising candidate and selected to the third (and final) phase of evaluation.

During the eSTREAM competition, LEX attracted a great deal of attention from cryptanalysts due to its simple structure, but nevertheless, only two attacks on the cipher were reported: A slide attack [23] requiring $2^{61}$ different IVs (each producing 20,000 keystream bytes), and a generic attack [17] requiring $2^{65.7}$ resynchronizations. Both attacks are applicable only against the original version of LEX presented in [6], but not against the tweaked version submitted to the second phase of eSTREAM [8]. In the tweaked version, the number of IVs used with a single key is bounded by $2^{32}$, and hence both attacks require too much data and are not applicable to the tweaked version.

In this paper we present an attack on LEX. The attack requires about $2^{36.3}$ bytes of key stream produced by the same key, possibly under different IVs. The time complexity of the attack is $2^{112}$ simple operations. Following a preliminary version of our attack, LEX was discarded from the final portfolio of eSTREAM.

Our attack is composed of three steps:

1. Identification of a special state: We focus our attention on pairs of AES encryptions whose internal states satisfy a certain difference pattern. While the probability of occurrence of the special pattern is $2^{-64}$, the pattern can be observed by a 32-bit condition on the output stream. Thus, the attacker repeats the following two steps for about $2^{32}$ cases which satisfy this 32 -bit condition.

2. Extracting information on the special state: By using the special difference pattern of the pair of intermediate values, and guessing the difference in eight more bytes, the attacker can retrieve the actual values of 16 internal state bytes in both encryptions.

3. Guess-and-Determine attack on the remaining unknown bytes: Using the additional known byte values, the attacker can mount a guess-anddetermine attack that retrieves the key using about $2^{112}$ simple operations in total. 
The second and the third steps of the attack use several observations on the structure of the AES round function and key schedule algorithm 1 One of them is the following, probably novel, observation:

Proposition 1. Denote the 128-bit subkey used in the $r$-th round of AES-128 by $k_{r}$, and denote the bytes of this subkey by an 4 -by-4 array $\left\{k_{r}(i, j)\right\}_{i, j=0}^{3}$. Then for every $0 \leq i \leq 3$ and $r$,

$$
k_{r}(i, 1)=k_{r+2}(i, 1) \oplus S B\left(k_{r+1}(i+1,3)\right) \oplus R C O N_{r+2}(i),
$$

where $S B$ denotes the SubBytes operation, $R C O N_{r+2}$ denotes the round constant used in the generation of the subkey $k_{r+2}$, and $i+1$ is replaced by 0 for $i=3$.

It is possible that the observations on the structure of AES presented in this paper can be used not only in attacks on LEX, but also in attacks on AES itself.

This paper is organized as follows: In Section 2 we briefly describe the structures of AES and LEX, and present the observations on AES used in our attack. In Section 3 we show that a specific difference pattern in the internal state can be partially detected by observing the output stream, and can be used to retrieve the actual value of 16 bytes of the internal state (in both encryptions). In Section 4 we leverage the knowledge of these 16 bytes into a complete key recovery attack that requires about $2^{112}$ simple operations. We give several additional observations that may be useful for further cryptanalysis of LEX in Section 5 . We conclude the paper in Section 6 .

\section{Preliminaries}

In this section we describe the structures of AES and LEX, and present the observations on AES used in our attack.

\subsection{Description of AES}

The advanced encryption standard 14] is an SP-network that supports key sizes of 128,192 , and 256 bits. As this paper deals with LEX which is based on AES128 , we shall concentrate the description on this variant and refer the reader to 22 for a complete detailed description of AES.

A 128-bit plaintext is treated as a byte matrix of size $4 \mathrm{x} 4$, where each byte represents a value in $G F\left(2^{8}\right)$. An AES round applies four operations to the state matrix:

- SubBytes (SB) - applying the same 8-bit to 8-bit invertible S-box 16 times in parallel on each byte of the state,

\footnotetext{
${ }^{1}$ We note that in 6] it was remarked that the relatively simple key schedule of AES may affect the security of LEX, and it was suggested to replace the AES subkeys by 1280 random bits. Our attack, which relies heavily on properties of the AES key schedule, would fail if such replacement was performed. However, some of our observations can be used in this case as well.
} 


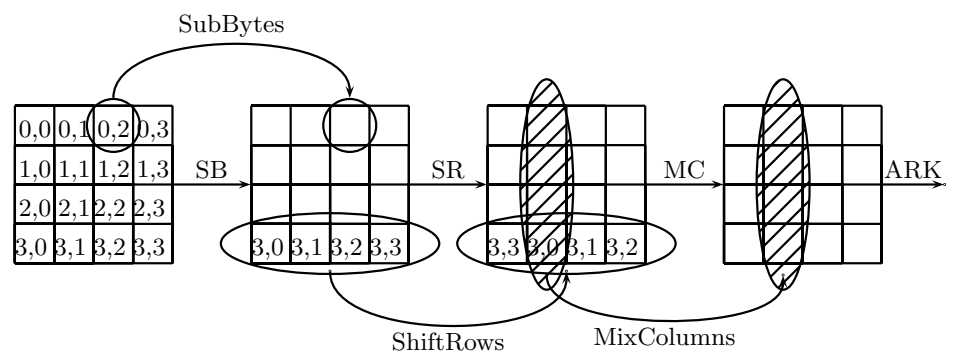

Fig. 1. An AES round

- ShiftRows (SR) - cyclic shift of each row (the $i$ 'th row is shifted by $i$ bytes to the left),

- MixColumns (MC) - multiplication of each column by a constant $4 \mathrm{x} 4$ matrix over the field $G F\left(2^{8}\right)$, and

- AddRoundKey (ARK) - XORing the state with a 128-bit subkey.

We outline an AES round in Figure 1. Throughout the paper we allow ourselves the abuse of notation $S B(x)$ to denote the application of the S-box to $x$ (whether it is one S-box when $x$ is 8 -bit value, or four times when $x$ is 32 -bit value). In the first round, an additional AddRoundKey operation (using a whitening key) is applied, and in the last round the MixColumns operation is omitted. We note that in LEX these changes to the first and last round are not applied.

AES-128, i.e., AES with 128-bit keys, has 10 rounds. For this variant, 11 subkeys of 128 bits each are derived from the key. The subkey array is denoted by $W[0, \ldots, 43]$, where each word of $W[\cdot]$ consists of 32 bits. The first four words of $W[\cdot]$ are loaded with the user supplied key. The remaining words of $W[\cdot]$ are updated according to the following rule:

- For $i=4, \ldots, 43$, do

- If $i \equiv 0 \bmod 4$ then $W[i]=W[i-4] \oplus S B(W[i-1] \lll 8) \oplus R C O N[i / 4]$,

- Otherwise $W[i]=W[i-1] \oplus W[i-4]$,

where $R C O N[\cdot]$ is an array of predetermined constants, and $\lll$ denotes rotation of the word by 8 bits to the left.

\subsection{Description of LEX}

For the ease of description, we describe only the tweaked version of LEX submitted to the second phase of eSTREAM [8]. The original version of LEX can be found in [6]. We note that our attacks can be easily adopted to the original version as well.

In the initialization step, the publicly known IV is encrypted by AES2 under the secret key $K$ to get $S=A E S_{K}(I V)$. Then, $S$ is repeatedly encrypted in the

\footnotetext{
${ }^{2}$ Actually, LEX uses a tweaked version of AES where the AddRoundKey before the first round is omitted, and the MixColumns operation of the last round is present. We allow ourselves the slight abuse of notations, for sake of clarity.
} 


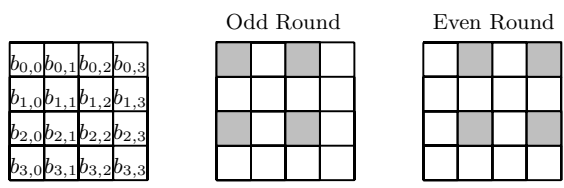

The gray bytes are the output bytes.

Fig. 2. Odd and Even Rounds of LEX

OFB mode of operation under $K$, where during the execution of each encryption, 32 bits of the internal state are leaked each round. These state bits compose the key stream of LEX. The state bytes used in the key stream are shown in Figure2, After 500 encryptions, another IV is chosen, and the process is repeated. After $2^{32}$ different IVs, the secret key is replaced 3

\subsection{Notations Used in the Paper}

As in [6], the bytes of each internal state during AES encryption, as well as the bytes of the subkeys, are denoted by a 4 -by- 4 array $\left\{b_{i, j}\right\}_{i, j=0}^{3}$, where $b_{i, j}$ is the $j$-th byte in the $i$-th row. For example, the output bytes in the even rounds are $b_{0,1}, b_{0,3}, b_{2,1}, b_{2,3}$.

\subsection{Observations on AES Used in Our Attack}

Throughout the paper we use several observations concerning AES.

Observation 1. For every non-zero input difference to the SubBytes operation, there are 126 possible output differences with probability $2^{-7}$ each (i.e., only a single input pair with the given difference leads to the specified output difference), and a single output difference with probability $2^{-6}$.

As a result, for a randomly chosen pair of input/output differences of the SubBytes operation, with probability $126 / 256$ there is exactly one unordered pair of values satisfying these differences. With probability $1 / 256$ there are two such pairs, and with probability $129 / 256$, there are no such pairs.

We note that while each ordered pair of input/output differences suggests one pair of actual values on average, it actually never suggests exactly one pair. In about half of the cases, two (or more) ordered pairs are suggested, and in the rest of the cases, no pairs are suggested. In the cases where two (or more) pairs are suggested, the analysis has to be repeated for each of the pairs. On the other hand, if no pairs are suggested, then the input/output differences pair is discarded as a wrong pair and the analysis is not performed at all. Hence,

${ }^{3}$ We note that in the original version of LEX, the number of different IVs used with a single key was not bounded. Following the slide attack presented in [23, the number of IVs used with each key was restricted. This restriction also prevents the attack suggested later in [17] which requires $2^{65.7}$ re-synchronizations. 
when factoring both events, it is reasonable to assume that each input/output differences pair suggests one pair of actual values.

Our attack uses this observation in situations where the attacker knows the input and output differences to some SubBytes operation. In such cases, using the observation she can deduce the actual values of the input and the output (for both encryptions). This can be done efficiently by preparing the difference distribution table [4 of the SubBytes operation, along with the actual values of the input pairs satisfying each input/output difference relation (rather than only the number of such pairs). In the actual attack, given the input and output differences of the SubBytes operation, the attacker can retrieve the corresponding actual values using a simple table lookup.

Observation 2. Since the MixColumns operation is linear and invertible, if the values (or the differences) in any four out of its eight input/output bytes are known, then the values (or the differences, respectively) in the other four bytes are uniquely determined, and can be computed efficiently.

The following two observations are concerned with the key schedule of AES. While the first of them is known (see [18]), it appears that the second was not published before.

Observation 3. For each $0 \leq i \leq 3$, the subkeys of AES satisfy the relations:

$$
\begin{aligned}
& k_{r+2}(i, 0) \oplus k_{r+2}(i, 2)=k_{r}(i, 2) . \\
& k_{r+2}(i, 1) \oplus k_{r+2}(i, 3)=k_{r}(i, 3) .
\end{aligned}
$$

Proof. Recall that by the key schedule, for all $0 \leq i \leq 3$ and for all $0 \leq j \leq 2$, we have $k_{r+2}(i, j) \oplus k_{r+2}(i, j+1)=k_{r+1}(i+1, j+1)$. Hence,

$$
\begin{gathered}
k_{r+2}(i, 0) \oplus k_{r+2}(i, 2)=\left(k_{r+2}(i, 0) \oplus k_{r+2}(i, 1)\right) \oplus\left(k_{r+2}(i, 1) \oplus k_{r+2}(i, 2)\right)= \\
k_{r+1}(i, 1) \oplus k_{r+1}(i, 2)=k_{r}(i, 2),
\end{gathered}
$$

and the second claim follows similarly.

Observation 4. For each $0 \leq i \leq 3$, the subkeys of AES satisfy the relation:

$$
k_{r+2}(i, 1) \oplus S B\left(k_{r+1}((i+1) \bmod 4,3)\right) \oplus R C O N_{r+2}(i)=k_{r}(i, 1),
$$

Proof. In addition to the relation used in the proof of the previous observation, we use the relation

$$
k_{r+2}(i, 0)=k_{r+1}(i, 0) \oplus S B\left(k_{r+1}((i+1) \bmod 4,3)\right) \oplus R C O N_{r+2}(i) .
$$

Thus,

$$
\begin{gathered}
k_{r+2}(i, 1) \oplus S B\left(k_{r+1}((i+1) \bmod 4,3)\right) \oplus R C O N_{r+2}(i)= \\
\left(k_{r+2}(i, 1) \oplus k_{r+2}(i, 0)\right) \oplus\left(k_{r+2}(i, 0) \oplus S B\left(k_{r+1}((i+1) \bmod 4,3)\right)\right. \\
\left.\oplus R C O N_{r+2}(i)\right)=k_{r+1}(i, 1) \oplus k_{r+1}(i, 0)=k_{r}(i, 1) .
\end{gathered}
$$

These two observations allow the attacker to use the knowledge of bytes of $k_{r+2}$ (and the last column of $k_{r+1}$ ) to get the knowledge of bytes in $k_{r}$, while "skipping" (some of) the values of $k_{r+1}$. 


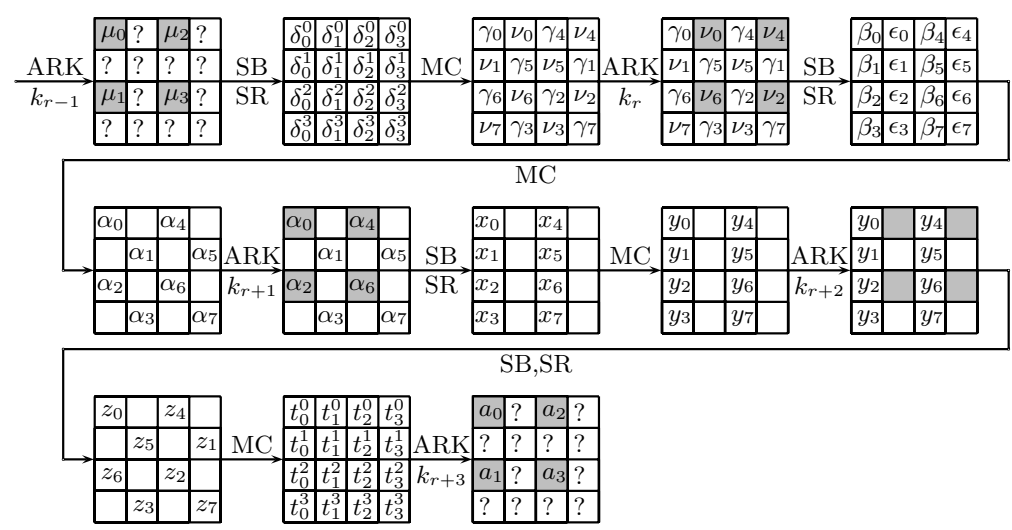

In gray we mark bytes whose value is known from the output key stream.

Fig. 3. The Special Difference Pattern (for Odd Rounds)

\section{Observable Difference Pattern in LEX}

Our attack is applicable when the special difference pattern starts either in odd rounds or in even rounds. For sake of simplicity of the description, we present the results assuming the difference pattern occurs in the odd rounds, and give in Appendix $\mathrm{A}$ the modified attack applicable when the difference pattern is observed in even rounds.

\subsection{Detecting the Difference Pattern}

Consider two AES encryptions under the same secret key, $K$. The special difference pattern corresponds to the following event: The difference between the intermediate values at the end of the $(r+1)$-th round is non-zero only in bytes $b_{0,0}, b_{0,2}, b_{1,1}, b_{1,3}, b_{2,0}, b_{2,2}, b_{3,1}$, and $b_{3,3}$. The probability of this event is $2^{-64}$. The pattern, along with the evolution of the differences in rounds $r, r+1, r+2$, and $r+3$, is presented in Figure 3 .

The difference pattern can be partially observed by a 32-bit condition on the output key stream: If the pattern holds, then all the four output bytes in round $r+2$ (bytes $b_{0,1}, b_{0,3}, b_{2,1}, b_{2,3}$ ) have zero difference.

Therefore, it is expected that amongst $2^{64}$ pairs of AES encryptions under the same key, one of the pairs satisfies the difference pattern, and about $2^{32}$ pairs satisfy the filtering condition. Thus, the following steps of the attack have to be repeated $2^{32}$ times on average (once for each candidate pair).

We note that if the special difference pattern is satisfied, then by the linearity of the MixColumns operation, there are only $255^{2}$ possible values for the difference in each of the columns before the MixColumns operation of round $r+1$ (denoted by $\beta$-s and $\epsilon$-s in Figure 3), and in each of the columns after the MixColumns operation of round $r+2$ (denoted by $t$-s in Figure 3 ). This property 
is used in the second step of the attack to retrieve the actual values of several state bytes.

\subsection{Using the Difference Pattern to Retrieve Actual Values of 16 Intermediate State Bytes}

In this section we show how the attacker can use the special difference pattern, along with a guess of the difference in eight additional bytes, in order to recover the actual values of 16 intermediate state bytes in both encryptions. We show in detail how the attacker can retrieve the actual value of byte $b_{0,0}$ of the state in the end of round $r$. The derivation of additional 15 bytes, which is performed in a similar way, is described briefly.

The derivation of the actual value of byte $b_{0,0}$ of the state at the end of round $r$ is composed of several steps (described also in Figure 4):

1. The attacker guesses the differences $\nu_{1}, \nu_{7}$ and applies the following steps for each such guess.

2. The attacker finds the difference in Column 0 before the MixColumns operation of round $r+1$, i.e., $\left(\beta_{0}, \beta_{1}, \beta_{2}, \beta_{3}\right)$. This is possible since the attacker knows the difference in Column 0 at the end of round $r+1$ (which is $\left(\alpha_{0}, 0, \alpha_{2}, 0\right)$ where $\alpha_{0}$ and $\alpha_{2}$ are known from the key stream), and since the AddRoundKey and the MixColumns operations are linear. By performing the inverse ShiftRows operation, the attacker can compute the output difference in byte $b_{0,0}$ after the SubBytes operation of round $r+1$.

3. Given the differences $\nu_{1}$ and $\nu_{7}$, there are $255^{2}$ possible differences after the MixColumns of round $\mathrm{r}$ in the leftmost column. Using the output bytes $b_{0,0}, b_{2,2}$ of round $r-1$, the attacker knows the difference in two bytes of the same column before the MixColumns operation. Hence, using Observation 2 (the linearity of the MixColumns operation), the attacker retrieves the difference in the whole column, both before and after the MixColumns operation, including the difference $\gamma_{0}$.

4. At this point, the attacker knows the input difference $\left(\gamma_{0}\right)$ and the output difference $\left(\beta_{0}\right)$ to the SubBytes operation in byte $b_{0,0}$ of round $r+1$. Hence, using Observation 1 (the property of the SubBytes operation), the attacker finds the actual values of this byte using a single table look-up. In particular, the attacker retrieves the actual value of byte $b_{0,0}$ at the end of round $r$.

The additional 15 bytes are retrieved in the following way:

1. The value of byte $b_{2,2}$ at the end of round $r$ is obtained in the same way using bytes $b_{0,2}, b_{2,0}$ of the output of round $r-1$ (instead of bytes $b_{0,0}, b_{2,2}$ ) and examining the third column (instead of the first one).

2. The value of bytes $b_{0,2}$ and $b_{2,0}$ at the end of round $r$ is found by examining $\alpha_{4}, \alpha_{6}$ (instead of $\left.\alpha_{0}, \alpha_{2}\right)$, guessing the differences $\nu_{3}, \nu_{5}$ (instead of $\left.\nu_{1}, \nu_{7}\right)$, and repeating the process used in the derivation of bytes $b_{0,0}, b_{2,2}$.

3. In a similar way, by guessing the differences $x_{1}, x_{3}, x_{5}, x_{7}$ and using the output bytes of round $r+3$, the attacker can retrieve the actual values of bytes $b_{0,0}, b_{0,2}, b_{2,0}$ and $b_{2,2}$ in the output of round $r+2$. 

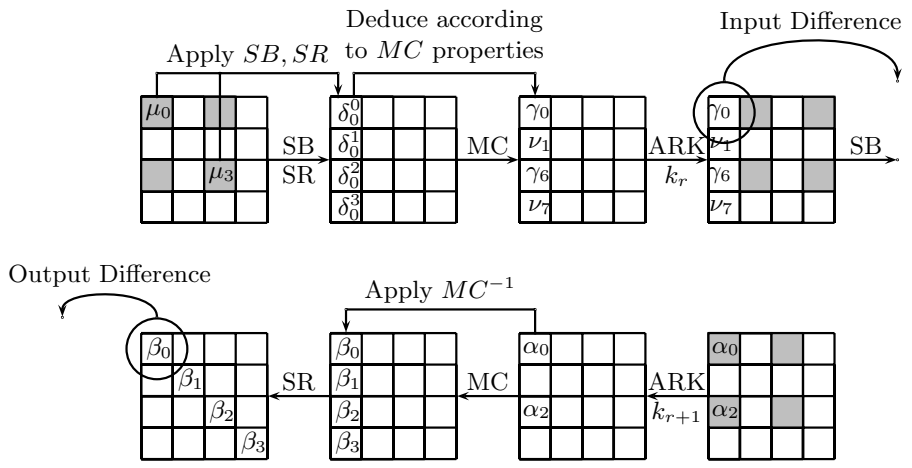

Fig. 4. Deducing the actual value of $b_{0,0}$ in the end of round $r$

4. Using the output of round $r$ and Observation 2, the attacker can obtain the differences $\alpha_{1}, \alpha_{3}, \alpha_{5}, \alpha_{7}$. Then, she can use the guessed differences $x_{1}, x_{3}$, $x_{5}, x_{7}$ and Observation 1 to obtain the actual values of bytes $b_{1,1}, b_{1,3}, b_{3,1}$ and $b_{3,3}$ at the end of round $r+1$.

5. Finally, using again the output of round $r$ and Observation 2, the attacker can obtain the differences $\epsilon_{1}, \epsilon_{3}, \epsilon_{5}, \epsilon_{7}$. Then, using the guessed differences $\nu_{1}, \nu_{3}, \nu_{5}, \nu_{7}$ and Observation 1 , the attacker can obtain the actual values of bytes $b_{1,0}, b_{1,2}, b_{3,0}$, and $b_{3,2}$ at the end of round $r$.

The bytes whose actual values are known to the attacker at this stage are presented in Figure 5 marked in gray.

\section{Retrieving the Key in the Special Cases}

The last step of the attack is a guess-and-determine procedure. Given the actual values of the 16 additional state bytes obtained in the second step of the attack, the entire key can be recovered using Observations 2 and 3 (properties of the MixColumns operation and of the key schedule algorithm of AES-128).

The deduction is composed of two phases. In the first phase, presented in Figure 5, no additional information is guessed. We outline in Appendix B the exact steps of the deduction. At the beginning of the second phase, presented in Figure 6, the attacker guesses the value of two additional subkey bytes. We outline in Appendix C the exact steps the attacker performs after guessing these two bytes. In both figures we use gray bytes to mark bytes which are known at the beginning of that deduction phase. Then, if a byte contains a number $i$ it means that this byte is computed in the $i$-th step of the deduction sequence.

Summarizing the attack, the attacker guesses 10 bytes of information ( 8 bytes of differences guessed in the second step of the attack, and 2 subkey bytes guessed in the third step of the attack), and retrieves the full secret key. Since all the operations used in the attack are elementary, the attack requires $2^{80}$ simple operations for each time the attack procedure is applied. Thus, as the attack 

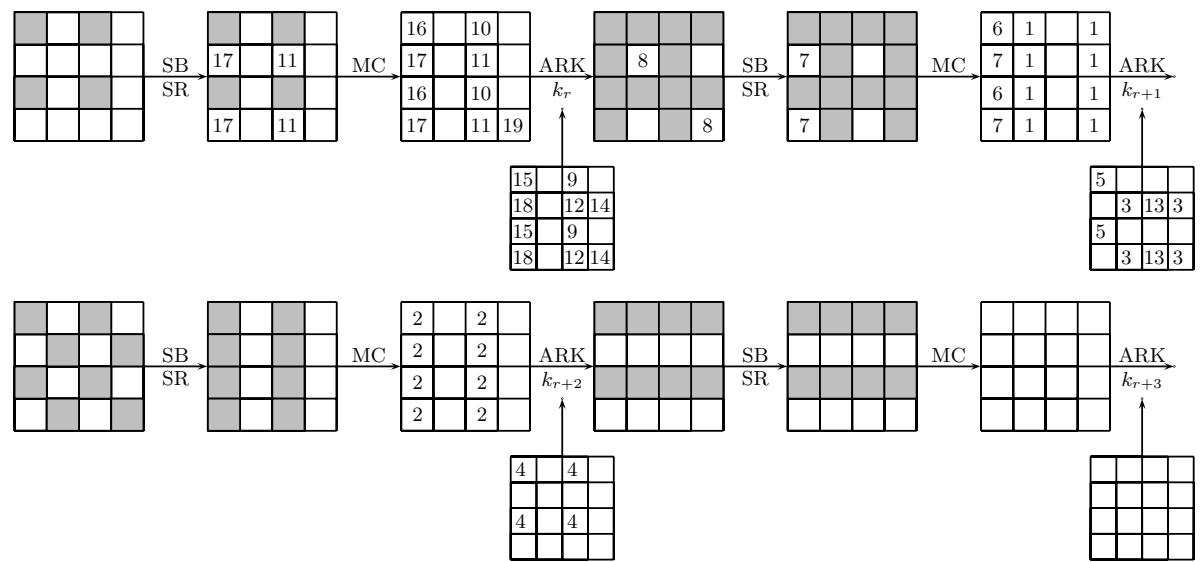

Gray boxes are bytes which are known.

Bytes marked with $i$, are bytes which are computed in step $i$.

Transition 9 is based on Observation 3.

Fig. 5. The First Phase of the Guess-and-Determine Attack on LEX (for Odd Rounds)

procedure is repeated $2^{32}$ times, the total running time of the attack is $2^{112}$ operations. Since the most time-consuming step of the attack is a guess-anddetermine procedure, it is very easy to parallelize the attack, and obtain a speed up equivalent to the number of used CPUs.

\subsection{Data Complexity of the Attack}

The attack is based on examining special difference patterns. Since the probability of occurrence of a special pattern is $2^{-64}$, it is expected that $2^{32.5}$ encryptions under the same key (possibly with different IVs) yield a single pair of encryptions satisfying the special pattern.

However, we note that the attack can be applied for several values of the starting round of the difference pattern. The attack presented above is applicable if $r$ is equal to $1,3,5$, or 7 , and a slightly modified version of the attack (presented in Appendix $\mathrm{A}$ is applicable if $r$ is equal to 0,2 , 4 , or 64 Hence, $2^{64} / 8=2^{61}$ pairs of encryptions are sufficient to supply a pair satisfying one of the eight possible difference patterns. These $2^{61}$ pairs can be obtained from $2^{31}$ AES encryptions, or equivalently, $2^{36.3}$ bytes of output key stream generated by the same key, possibly under different IVs.

\footnotetext{
${ }^{4}$ We note that while the attack considers five rounds of the encryption (rounds $r-1$ to $r+3$ ), it is not necessary that all the five rounds are contained in a single AES encryption. For example, if $r=7$ then round $r+3$ considered in our attack is actually round 0 of the next encryption. The only part of the attack which requires the rounds to be consecutive rounds of the same encryption is the key schedule considerations. However, in these considerations only three rounds (rounds $r$ to $r+2$ ) are examined.
} 

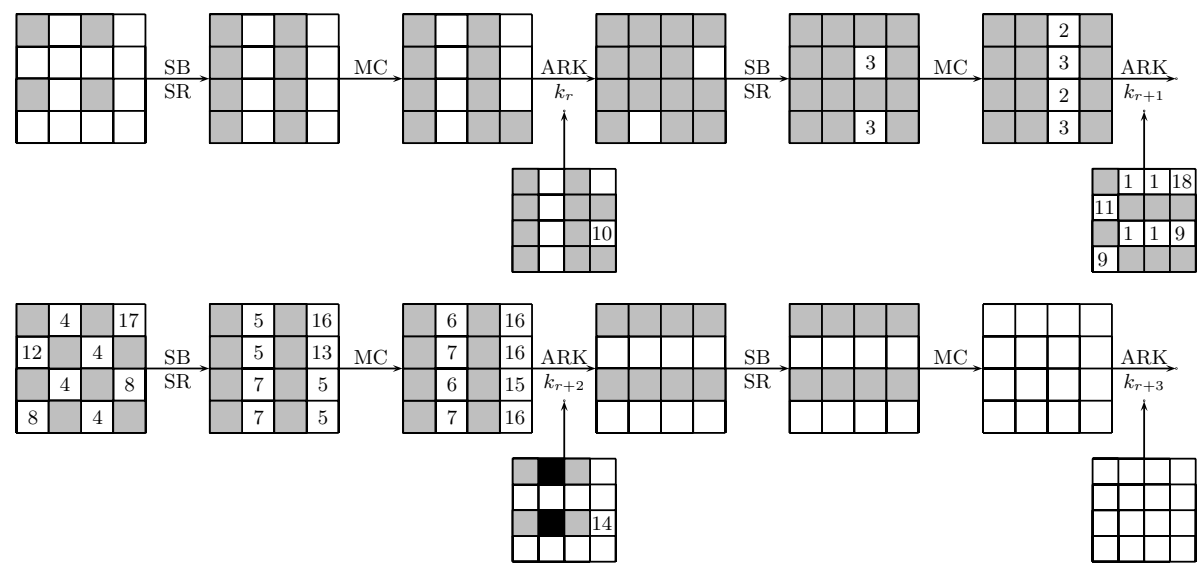

Gray boxes are bytes which are known.

Black boxes are the two bytes guessed in this phase.

Bytes marked with $i$, are bytes which are computed in step $i$ of the second phase.

Fig. 6. The Second Phase of the Guess-and-Determine Attack on LEX (for Odd Rounds)

\section{Further Observations on LEX}

In this section we present several observations on the structure of LEX that may be helpful in further cryptanalysis of the cipher.

\subsection{Sampling Resistance of LEX}

One of the main advantages of LEX, according to the designers (see [6], Section 1 ), is the small size of its internal state allowing for a very fast key initialization (a single AES encryption). It is stated that the size of the internal state (256 bits) is the minimal size assuring resistance to time-memory-data tradeoff attacks.

Time-memory-data tradeoff (TMDTO) attacks [2910 20] are considered a serious security threat to stream ciphers, and resistance to this class of attacks is a mandatory in the design of stream ciphers (see, for example, [16]). A cipher with an $n$-bit key is considered (certificationally) secure against TMDTO attacks if any TMDTO attack on the cipher has either data, memory, or time complexity of at least $2^{n}$.

In order to ensure security against conventional TMDTO attacks trying to invert the function (State $\rightarrow$ Key Stream), it is sufficient that the size of the internal state is at least twice the size of the key [10]. LEX satisfies this criterion (the key size is 128 bits and the size of the internal state is 256 bits). As a result, as claimed by the designers (see [6], Sections 3.2 and 5), the cipher is secure with respect to TMDTO attacks. 
However, as observed in [1], having the size of the internal state exactly twice larger than the key length is not sufficient if the cipher has a low sampling resistance. Roughly speaking, a cipher has a sampling resistance of $2^{-t}$, if it is possible to list all internal states which lead to some $t$-bit output string efficiently. In other words, if it is possible to find a (possibly special) string of $t$ bits, whose "predecessor" states are easily computed, then the cipher has sampling resistance of at most $2^{-t}$.

It is easy to see that LEX has maximal sampling resistance of $2^{-32}$, as out of the 256 bits of internal state, 32 bits are output directly every round. As a result, using the attack algorithm presented in [10, it is possible to mount a TMDTO attack on LEX with data complexity $2^{88}$, and time and memory complexities of $2^{112}$. Hence, LEX provides only 112-bit security with respect to TMDTO attacks.

\subsection{Loss of Entropy in the Initialization of LEX}

The first step in the initialization of LEX is the encryption of $I V$ by AES under the secret key $K$. When considering $A E S_{K}(I V)$ as a function of $K$, one can easily see that under reasonable randomness assumptions on AES, this function is a random function of the key $K$. As a result, the first internal state $S$ used in LEX, does not contain 128 bits of entropy, even when the IV has full entropy. Actually, the expected number of possible $S$ 's for a given $I V$ is about $63 \%$ of all possible values, i.e., about $2^{127.3}$ possible $S$ 's.

Even though our attack does not use this observation, it might still be used in attacks which rely on entropy. Especially, the variant of [15] of time-memory-data tradeoff attacks (trying to invert the function $(k e y, I V) \rightarrow$ keystream) might use this observation by trying to invert the function $(k e y, S) \rightarrow$ keystream.

\subsection{Analysis of the Submitted Reference Implementation of the Original (Untweaked) Version of LEX}

After communicating a preliminary version of our attack, we received a request to discuss the implementation of the original (untweaked) version of LEX submitted to eSTREAM. According to a claim made in [3] and verified later by us, the submitted code of the untweaked LEX outputs different bytes than intended and specified (specifically, in the even rounds, $b_{1,1}, b_{1,3}, b_{3,1}$ and $b_{3,3}$ are given as the key stream). Of course, this seems like an unintended typo made in the submission pack (as the fact that it was corrected in the tweaked submission of LEX).

It appears that this variant is much weaker than the intended cipher: First, given the difference in the key stream corresponding to an even round of AES and the consecutive odd round, the difference in two full columns (i.e., four additional internal bytes) can be found easily, without any assumption on the difference between the states. Second, it is possible to devise a simple meet-inthe-middle attack which uses only 256 bits of output stream and retrieves the secret key using $2^{112}$ simple operations. 
The difference between the security of the intended version and that of the actual implementation emphasizes the importance of verifying the implementations of cryptographic primitives very carefully. This importance was first observed in [12] with respect to public key encryption, and adopted in [5] to the symmetric key scenario. While differential fault analysis assumes that the attacker can access both a faulty implementation and a regular implementation, our observations are valid when the attacker has to attack only the faulty implementation.

\section{Summary and Conclusions}

In this paper we presented a new attack on the LEX stream cipher. We showed that there are special difference patterns that can be easily observed in the output key stream, and that these patterns can be used to mount a key recovery attack.

The attack uses a total of $2^{36.3}$ bytes of key stream produced by a single key (possibly under different IVs) and takes $2^{112}$ simple operations to implement.

Our results show that for constructions based on the Goldreich-Levin approach (i.e., PRNGs based on pseudo-random permutations), the pseudorandomness of the underlying permutation is crucial to the security of the resulting stream cipher. In particular, a small number of rounds of a (possibly strong) block cipher cannot be considered random in this sense, at least when a non-negligible part of the internal state is extracted.

\section{References}

1. Babbage, S.H., Dodd, M.: Specification of the Stream Cipher Mickey 2.0, submitted to eSTREAM (2006), http://www.ecrypt.eu.org/stream/p3ciphers/mickey/mickey_p3.pdf

2. Babbage, S.H: Improved "exhaustive search" attacks on stream ciphers. In: IEE European Convention on Security and Detection, IEE Conference publication, vol. 408, pp. 161-165. IEE (1995)

3. Bernstein, D.J.: Personal communication (2008)

4. Biham, E., Shamir, A.: Differential Cryptanalysis of the Data Encryption Standard. Springer, Heidelberg (1993)

5. Biham, E., Shamir, A.: Differential Fault Analysis of Secret Key Cryptosystems. In: Kaliski Jr., B.S. (ed.) CRYPTO 1997. LNCS, vol. 1294, pp. 513-525. Springer, Heidelberg (1997)

6. Biryukov, A.: The Design of a Stream Cipher LEX. In: Biham, E., Youssef, A.M. (eds.) SAC 2006. LNCS, vol. 4356, pp. 67-75. Springer, Heidelberg (2007)

7. Biryukov, A.: A New 128-bit Key Stream Cipher LEX, ECRYPT stream cipher project report 2005/013, http://www.ecrypt.eu.org/stream

8. Biryukov, A.: The Tweak for LEX-128, LEX-192, LEX-256, ECRYPT stream cipher project report 2006/037, http://www.ecrypt.eu.org/stream 
9. Biryukov, A., Mukhopadhyay, S., Sarkar, P.: Improved Time-Memory Tradeoffs with Multiple Data. In: Preneel, B., Tavares, S. (eds.) SAC 2005. LNCS, vol. 3897, pp. 245-260. Springer, Heidelberg (2006)

10. Biryukov, A., Shamir, A.: Cryptanalytic Time/Memory/Data Tradeoffs for Stream Ciphers. In: Okamoto, T. (ed.) ASIACRYPT 2000. LNCS, vol. 1976, pp. 1-13. Springer, Heidelberg (2000)

11. Blum, M., Micali, S.: How to Generate Cryptographically Strong Sequences of Pseudo-Random Bits. SIAM Journal of Computation 13(4), 850-864 (1984)

12. Boneh, D., DeMillo, R.A., Lipton, R.J.: On the Importance of Checking Cryptographic Protocols for Faults (Extended Abstract). In: Fumy, W. (ed.) EUROCRYPT 1997. LNCS, vol. 1233, pp. 37-51. Springer, Heidelberg (1997)

13. Daemen, J., Rijmen, V.: AES Proposal: Rijndael, NIST AES proposal (1998)

14. Daemen, J., Rijmen, V.: The design of Rijndael: AES - the Advanced Encryption Standard. Springer, Heidelberg (2002)

15. Dunkelman, O., Keller, N.: Treatment of the Initial Value in Time-Memory-Data Tradeoff Attacks on Stream Ciphers. Information Processing Letters 107(5), 133137 (2008)

16. ECRYPT, Call for Stream Cipher Primitives, version 1.3 (April 12, 2005), http://www.ecrypt.eu.org/stream/call/

17. Englund, H., Hell, M., Johansson, T.: A Note on Distinguishing Attacks. In: Preproceedings of State of the Art of Stream Ciphers workshop (SASC 2007), Bochum, Germany, pp. 73-78 (2007)

18. Ferguson, N., Kelsey, J., Lucks, S., Schneier, B., Stay, M., Wagner, D., Whiting, D.: Improved Cryptanalysis of Rijndael. In: Schneier, B. (ed.) FSE 2000. LNCS, vol. 1978, pp. 213-230. Springer, Heidelberg (2001)

19. Goldreich, O., Levin, L.A.: A Hard-Core Predicate for all One-Way Functions. In: Proceedings of 21st STOC, pp. 25-32. ACM, New York (1989)

20. Golic, J.D.: Cryptanalysis of Alleged A5 Stream Cipher. In: Fumy, W. (ed.) EUROCRYPT 1997. LNCS, vol. 1233, pp. 239-255. Springer, Heidelberg (1997)

21. Håstad, J., Näslund, M.: BMGL: Synchronous Key-stream Generator with Provable Security. NESSIE project (submitted, 2000), http://www.nessie.eu.org

22. National Institute of Standards and Technology, Advanced Encryption Standard, Federal Information Processing Standards Publications No. 197 (2001)

23. Wu, H., Preneel, B.: Attacking the IV Setup of Stream Cipher LEX, ECRYPT stream cipher project report 2005/059, http://www.ecrypt.eu.org/stream

\section{A Special Difference Pattern Starting with an Even Round}

In this section we present the modified version of the attack that can be applied if the special difference pattern occurs in the even rounds. The first two steps of the attack (observing the difference pattern and deducing the actual values of 16 additional bytes of the state) are similar to the first two steps of the attack presented in Section 3. The known byte values after these steps are presented in Figure 7. marked in gray. The third step of the attack is slightly different due to the asymmetry of the key schedule, and Observation 4 is used in this step along with Observations 2 and 3. The two phases of this step are presented in 


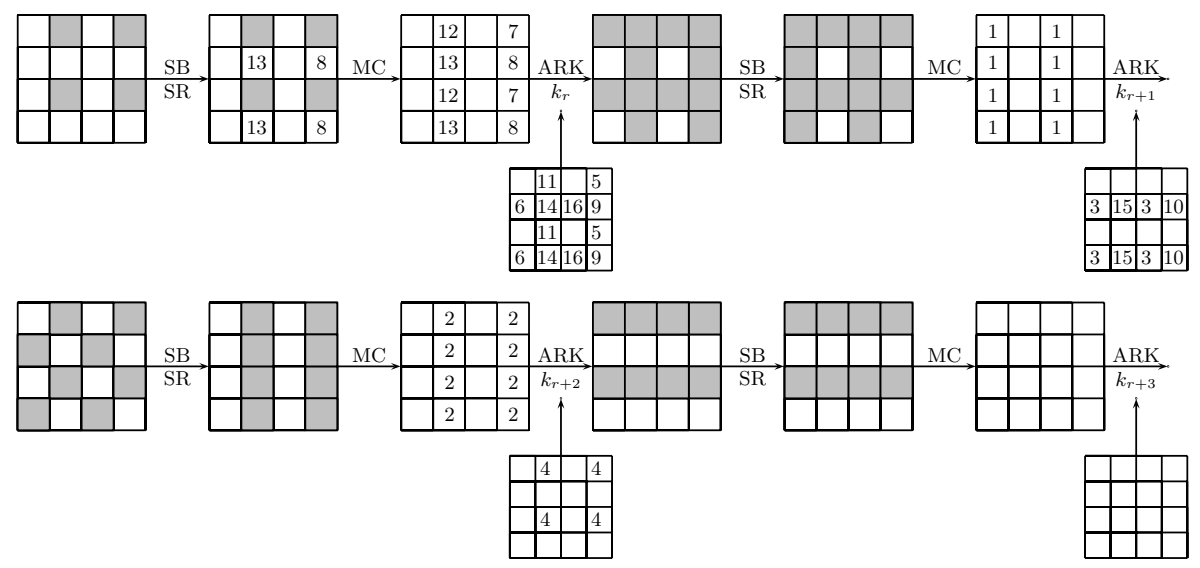

Gray boxes are bytes which are known.

Bytes marked with $i$, are bytes which are computed in step $i$.

Step 5 is based on Observation 3, and step 11 is based on Observation 4.

Fig. 7. The First Phase of the Guess-and-Determine Attack on LEX (in Even Rounds)

Figures 7 and 8 . The overall time complexity of the attack is $2^{112}$ operations, like in the case of a difference pattern in the odd rounds.

\section{B Detailed Description of the Steps in the First Deduction Phase}

In this section we present the exact deduction steps done during the first phase depicted in Figure 5. The numbers of the steps correspond to the numbers in the figure.

1. The application of MixColumns in round $r+1$ on two columns (second and fourth) gives these bytes.

2. The application of MixColumns in round $r+2$ on two columns (first and third) gives these bytes.

3. The knowledge of the value of four bytes before the XOR with the subkey $k_{r+1}$ and after the XOR, gives the value of the subkey in these bytes.

4. The knowledge of the value of four bytes before the XOR with the subkey $k_{r+2}$ and after the XOR, gives the value of the subkey in these bytes.

5 . By the key schedule of AES, the knowledge of byte $(0,0)$ of the subkey $k_{r+2}$ and byte $(1,3)$ of the subkey $k_{r+1}$ gives the value of byte $(0,0)$ of the subkey $k_{r+1}$. Similarly, the knowledge of byte $(2,0)$ of the subkey $k_{r+2}$ and byte $(3,3)$ of the subkey $k_{r+1}$ gives the value of byte $(2,0)$ of the subkey $k_{r+1}$.

6. These two bytes are the XOR of the two subkey bytes found in the previous step and known bytes. 

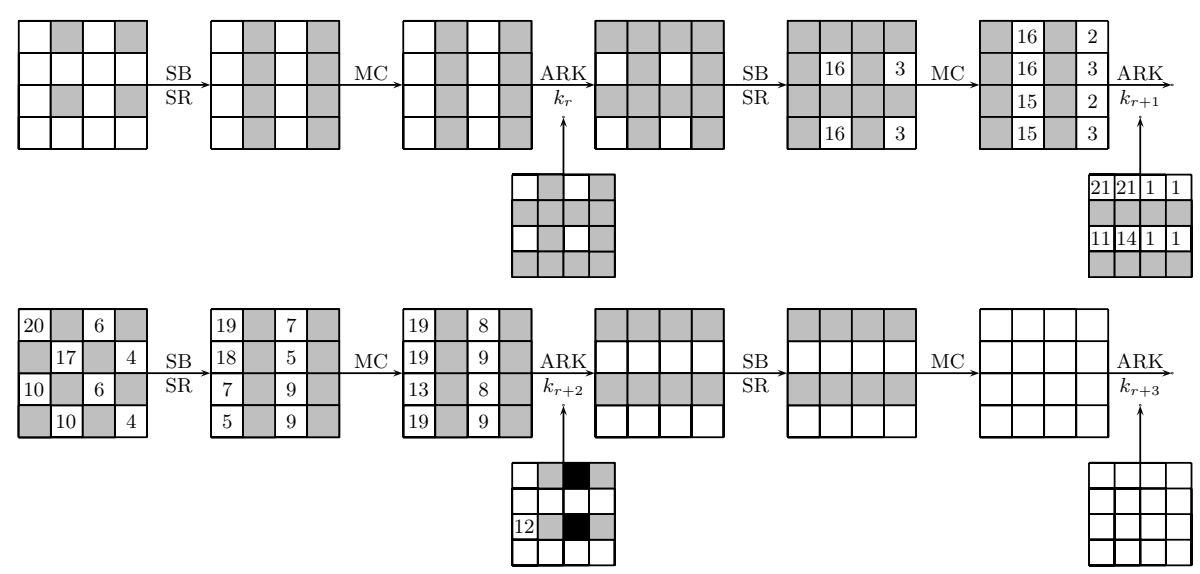

Gray boxes are bytes which are known.

Black boxes are the two bytes guessed in this phase.

Bytes marked with $i$, are bytes which are computed in step $i$ of the second phase.

Fig. 8. The Second Phase of the Guess-and-Determine Attack on LEX (in Even Rounds)

7. Applying Observation 2 to the first column in the MixColumns operation of round $r+1$ gives these four bytes.

8. The two bytes after the SubBytes and ShiftRows operation are just computed backwards.

9. These bytes are computed using the four bytes found in Step 4, and the application of Observation 3 .

10. These bytes are computed by XORing the subkey bytes found in the previous step with known values.

11. Applying Observation 2 to the third column in the MixColumns operation of round $r$ gives these four bytes.

12. The input and output of the AddRoundKey operation of round $k_{r}$ in these two bytes is known, and allows retrieving these two subkey bytes.

13. By the key schedule of AES, the knowledge of bytes $(1,1)$ and $(3,1)$ of the subkey $k_{r+1}$ and bytes $(1,2)$ and $(3,2)$ of the subkey $k_{r}$ gives the values of bytes $(1,2)$ and $(3,2)$ of the subkey $k_{r+1}$, respectively.

14. By the key schedule of AES, the knowledge of bytes $(1,2)$ and $(3,2)$ of the subkey $k_{r}$ and bytes $(1,3)$ and $(3,3)$ of the subkey $k_{r+1}$ gives the values of bytes $(1,3)$ and $(3,3)$ of the subkey $k_{r}$, respectively.

15. By the key schedule of AES, the knowledge of byte $(0,0)$ of the subkey $k_{r+1}$ and byte $(1,3)$ of the subkey $k_{r}$ gives the value of byte $(0,0)$ of the subkey $k_{r}$. Similarly, the knowledge of byte $(2,0)$ of the subkey $k_{r+1}$ and byte $(3,3)$ of the subkey $k_{r}$ gives the value of byte $(2,0)$ of the subkey $k_{r}$.

16. These bytes are computed by XORing the subkey bytes found in the previous step with known values. 
17. Applying Observation 2 to the first column in the MixColumns operation of round $r$ gives these four bytes.

18. These bytes are the XOR of the bytes found in the previous step with known bytes.

19. This byte is the XOR of one of the bytes found in Step 14 and a byte found in Step 8.

\section{Detailed Description of the Steps in the Second Deduction Phase}

In this section we present the exact deduction steps performed during the second phase depicted in Figure 6. The numbers of the steps correspond to the numbers in the figure.

1. Using the key schedule algorithm, it is possible to deduce four bytes of $k_{r+1}$ (each is the XOR of two known bytes in $k_{r+2}$ ).

2. Decrypting two known bytes using two of the subkey words found in Step 1 gives these two bytes.

3. Applying Observation 2 to the third column in the MixColumns operation of round $r+1$.

4. These four bytes are computed by the XOR of known state bytes and subkey bytes (in $k_{r+1}$ ).

5. These four bytes are the application of the SubBytes and ShiftRows operations on the bytes found in the previous step.

6 . These bytes are the XOR of known bytes and the subkey bytes that were guessed.

7. Applying Observation 2 to the second column in the MixColumns operation of round $r+2$ gives these four bytes.

8. These two bytes are found by applying the inverse ShiftRows and SubBytes operations to two of the bytes found in the previous step.

9. These two subkey bytes are computed as the XOR of the corresponding bytes before and after the AddRoundKey operation of round $r+1$.

10. By the key schedule of AES, the knowledge of byte $(3,0)$ of the subkey $k_{r+1}$ and byte $(3,0)$ of the subkey $k_{r}$ gives the value of byte $(2,3)$ of the subkey $k_{r}$.

11. By the key schedule of AES, the knowledge of bytes $(1,0)$ and $(2,3)$ of the subkey $k_{r+1}$ gives the value of byte $(1,0)$ of the subkey $k_{r+1}$.

12. This byte is the XOR of a known state byte with the subkey byte found in the previous step.

13. This byte is computed by applying the SubBytes and ShiftRows operations to the byte found in the previous step.

14. By the key schedule of AES, the knowledge of byte $(2,2)$ of the subkey $k_{r+2}$ and byte $(2,3)$ of the subkey $k_{r+1}$ gives the value of byte $(2,3)$ of the subkey $k_{r+2}$.

15. This byte is the XOR of a known state byte with the subkey byte found in the previous step. 
16. This byte is the partial decryption of a known byte by the byte found in the previous step.

17. Applying Observation 2 to the fourth column in the MixColumns operation of round $r+2$ gives these four bytes.

18. This byte is found by applying the inverse ShiftRows and SubBytes operations to one of the bytes found in the previous step.

19. This byte is the partial decryption of a known byte by the byte found in the previous step. 